\title{
Analyzing uplink capacity of partially overlapping channel based WLANs using a hyper-receiver
}

\author{
Muhammad I. Majid, Muhammad A. Imran, Reza Hoshyar \\ Centre for Communications and Systems Research \\ University of Surrey, Guildford, \\ GU2 7XH, United Kingdom \\ Email: \{m.majid, m.imran, r.hoshyar\}@ surrey.ac.uk
}

\begin{abstract}
Spectrum allocation within the fixed unlicensed band affects performance of wireless networks. Fundamental limitations of spectral efficiency on capacity of wireless local area networks (WLANs) hence needs to be studied. Recently, it was shown that the performance can be increased when both nonoverlapping and partially overlapping channels are used. Unlike previous studies, this is the first known attempt on analysis of information theoretic capacity for partially-overlapping channels, as characterized by IEEE 802.11b-type systems using a hyperreceiver. Using Marcĕnko-Pastur Law distribution, capacity is approximated for such systems. We implement this by proposing a novel channel model for our analysis. The results conform to Monte Carlo simulations, with an approximate mean error of $5 \%$. Fundamental tradeoffs of channel interference and power gain are discussed. We further explore the effect of access point density and find that systems based on partially overlapping channels perform well in high density deployments. We also compute capacity for a range of channel overlaps and conclude that for high density, rates close to capacity are achievable for a channel overlap of $90 \%$ and above.
\end{abstract}

\section{INTRODUCTION}

Many telecom operators have deployed IEEE $802.11 \mathrm{~b}$ wireless local area networks (WLANs) as an economical and versatile solution to meet the growing needs of the digital customer. It has applications in citywide wireless deployments [1] and body area networks [2], amongst others. The IEEE 802.11b standard recommends 13 operating channels in Europe, spread over a bandwidth of $85 \mathrm{MHz}$, of which three are non-overlapping while the remaining overlap in the frequency domain. Only the three non-overlapping channels are used in current systems as verified by access point (AP) vendors [3]. This is to avoid contention during transmission and reduce interference in the unlicensed Industrial Scientific and Medical (ISM) band. The remaining ten channels partially overlap in the frequency domain, they are referred to as partially overlapping channels (POCs). Conventionally, POCs have not been popular. However, given the fact that ISM band is a limited resource, using only three channels is spectrally inefficient, and hence waste system resources. Moreover, utilization of channels has become important as the density of wireless systems grow. We present an asymptotic capacity analysis for large number of $802.11 \mathrm{~b}$ type WLAN cells and hence evaluate an upper bound assuming a hyper-receiver [4]. Further, we

The first author is a Commonwealth Scholar.

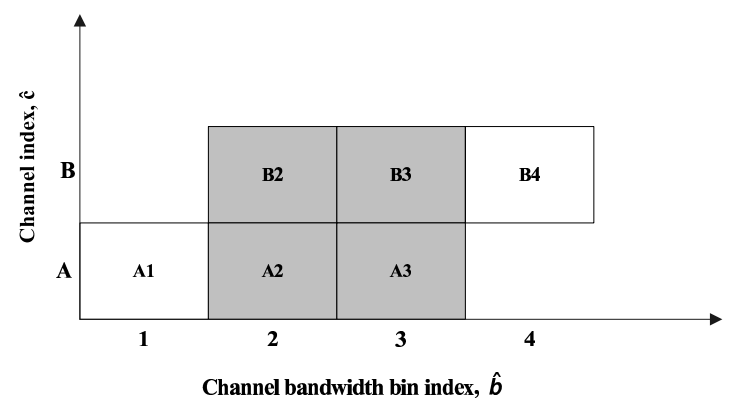

B2 Power transmitted over fully overlapping bin for user transmitting in channel B, with bandwidth constrained by bin index 2

A1 Power transmitted over non-overlapping bin for user transmitting in channel A, with bandwidth constrained by bin index 1

Fig. 1. Basic concept of bandwidth division. Vertical axis represent the channel indices. Height of bins represents the power spectral density of channel bins which is a constant. The area with any bin is a measure of maximum power that can be transmitted within that bin.

introduce bandwidth variation for variable AP density in our model for systems comparison. This work is the first known attempt on information theoretic capacity analysis of IEEE 802.11b-based POCs, assuming a hyper-receiver.

The rest of the paper is organized as follows: In Section II we review the related work on POCs and hyper-receivers in context of linear cell networks. In Section III, we introduce the basic model using division of bandwidth into bins of equal size. In Section IV, we analyze capacity approximation based on Marcěnko Pastur Law and validate it using Monte Carlo simulations. In Section V, we explain the affect of AP density on POCs operating in different bandwidth regimes and identify the suitable region of operation. A comparison of analytical and simulated capacity is presented. Finally, in Section VI, we conclude.

\section{RELATED WORK}

An 802.11b outdoor cellular network was studied in [5]. The concept of a hyper-receiver was introduced in Wyner's Gaussian Cellular Multiple Access Channel (GCMAC) model [4]. It assumed that a central processor had instantaneous access to codebooks of all users. Flat Rayleigh fading was added to GCMAC by Somekh and Shamai [6]. Letzepis [7] 


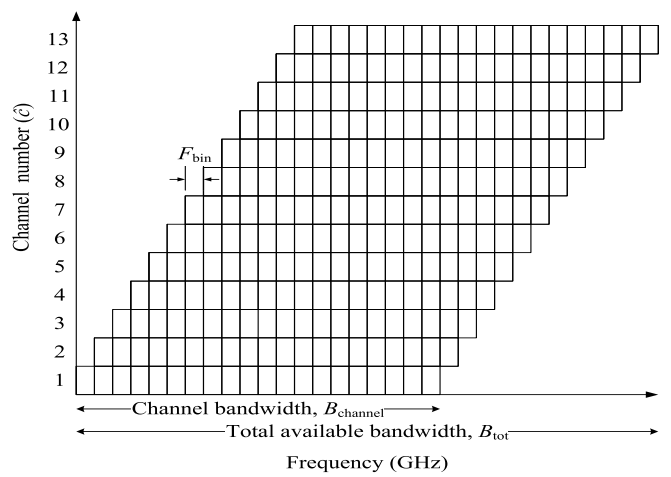

Fig. 2. IEEE $802.11 \mathrm{~b}$ type channel structure with modelling parameters for the partial overlap channel model.

modified it further to model free space pathloss incurred by each transmitted signal. More recently, interest has been shown in POCs-based mesh-networks [8]-[10]. Mishra et al. [11] and Akl [12] proposed algorithms to evaluate the throughput of both non-overlapping channels and POCs. Heuristics was used to solve their channel assignment problem. Such solutions, however are not always optimal. A more fundamental insight into the partial overlap nature and its affect on capacity, in low and high signal to noise ratios (SNRs) is required. We aim to get such an insight using the model described.

\section{SySTEM MODEL}

\section{A. System Assumptions}

The system consists of a linear array of $N$ cells, uniformly spaced over a span $D$. APs and users are co-located at the center of each cell. Per-cell sum-rate capacity for uplink of the system is evaluated. APs are assigned POCs with assignment as per the sequence: $1,2,3, \ldots, 12,13,1,2,3, \ldots$. All the received signals are jointly decoded and receiver is assumed to be aware of channel parameters. External interference is treated as AWGN. We assume single user per cell $(K=1)$ in order to understand the complicated nature of the partial overlap channel structure. We further assume that an infrastructure based WLAN with interconnected APs is equivalent to a cellular system (as in Section II). A hyper-receiver is employed by which each AP is aware of codebooks of all users. This provides capacity achieving strategy [6]. We believe such a complex receiver will be available in the near future.

A synopsis of the channel model assumptions is presented. The channel structure adopted is as per the IEEE $802.11 \mathrm{~b}$ standards (13 POCs, and system bandwidth of $85 \mathrm{MHz}$ ). Keeping the system bandwidth constant, the channel bandwidth is varied. The channel is partitioned into frequency bins of constant power spectral density. The total frequency bins within a given channel bandwidth have a sum power constraint. This constraint is equal to the transmit power per channel. Further, since all channels occupy the same bandwidth, maximum transmit power for all channel users is the same.

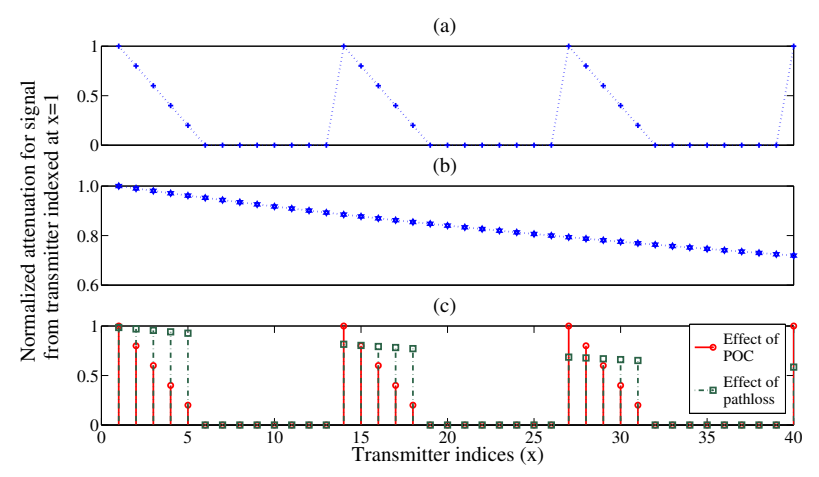

Fig. 3. Effect on channel gain of a linear AP array (40 cells) due to (a) POC, (b) pathloss, (c) combined effect of pathloss and POC are shown. Here $B_{\text {channel }} \cong 0.25 B_{\text {tot }}$ for a free space path loss IEEE 802.11 b type system $\left(\tilde{C}=13, B_{\text {tot }} \cong 85 \mathrm{MHz}\right)$.

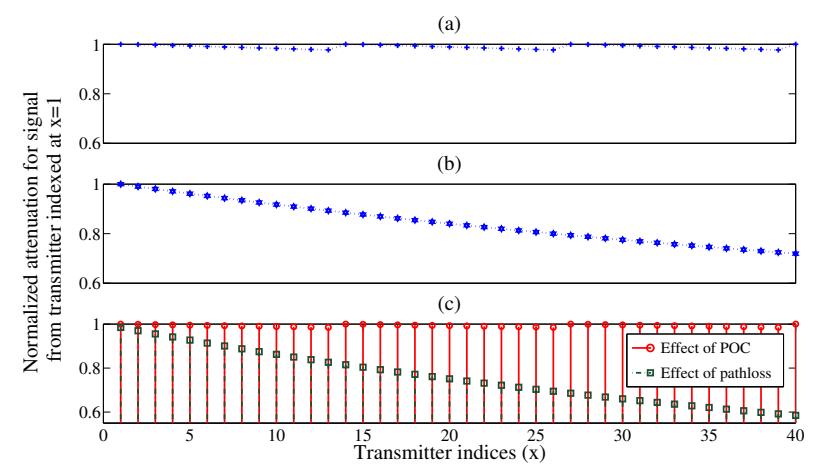

Fig. 4. Effect on channel gain of a linear AP array (40 cells) due to (a) POC, (b) pathloss, (c) combined effect of pathloss and POC are shown. Here $B_{\text {channel }} \cong B_{\text {tot }}$ for a free space path loss IEEE 802.11 b type system $\left(\tilde{C}=13, B_{\text {tot }} \cong 85 \mathrm{MHz}\right)$.

\section{B. Model Description}

We use partitioning of channel bandwidth into bins of constant power spectral density. This is done to facilitate the analysis of channel overlap. The frequency bins are used to synthesize the total bandwidth so the system only consists of fully overlapping or non overlapping bins as shown in Fig. 1. More specifically, we consider an IEEE 802.11 b type channel spectrum as shown in Fig. 2. The vertical axis represents channel indices and the horizontal axis shows spectrum allocation for each channel in $\mathrm{GHz}$. We assume that each channel bandwidth can be subdivided into orthogonal narrowband frequency bins occupying $F_{\text {bin }}$ expressed in $\mathrm{Hz}$. Each channel user transmits over a channel bandwidth, $B_{\text {channel }}$ which is in $\mathrm{Hz}$ and consists of $\frac{B_{\text {channel }}}{F_{\text {bin }}}$ bins. The total channels $\tilde{C}$ are indexed with $\tilde{c}$. The total system bandwidth is $B_{\text {tot }} \mathrm{Hz}$. Analytically the total number of frequency bins per channel can be represented as:

$$
\frac{B_{\text {channel }}}{F_{\text {bin }}}=\tilde{B}-(\tilde{C}-1)
$$

where $\tilde{B}$ is the number of bins that can be accommodated within the total system bandwidth. By using (1), it is possible 
to evaluate the channel-to-total-system bandwidth ratio for a POCs based system:

$$
\frac{B_{\text {channel }}}{B_{\text {tot }}}=\frac{\frac{B_{\text {channel }}}{F_{\text {bin }}}}{\frac{B_{\text {channel }}}{F_{\text {bin }}}+\tilde{C}-1} .
$$

From (2) we can compute different values for $B_{\text {channel }}$ assuming fixed $B_{\text {tot }}$ to explore different overlapping regions in a POCs based system.

\section{CAPACITY ANALYSIS FOR POC MODEL WITH FADING}

We model POCs (as discussed in Mishra et al. [11] and Akl [12]), to compute information theoretic capacity of $802.11 \mathrm{~b}$ type WLANs. Letzepis [7] pathloss model for GCMAC is modified to cater for the POC structure, an inherent feature of WLANs. In Section IV-A, we describe approximation based on Marčenko-Pastur Law which leads to numerical computation of capacity. Capacity of the partial overlapping structure with flat fading is validated by using Monte Carlo simulations in Section IV-B.

\section{A. Capacity approximation using Marčenko-Pastur Law}

To measure the spectral efficiency of POCs based systems, we approximate per-cell sum capacity using tools of free probability, as done in Letzepis [7]. First, we define $\mathbf{G}$ as a $N \times N$ complex Gaussian matrix which has Independent and Identically Distributed (i.i.d) entries, Complex and Circularly Symmetric (c.c.s.) Gaussian distributed with zero-mean and variance, $\frac{1}{N}$. $G$ is used to define model multipath fading. Further, let $\boldsymbol{\Sigma}_{\mathbf{Q}}$ be a deterministic $N \times N$ matrix whose entries represent the channel attenuation due to non-fading elements of channel matrix. $\boldsymbol{\Sigma}_{\mathbf{Q}}$ is hence used to evaluate limiting eigenvalue distributions of the covariance of channel matrix $\mathbf{H H}^{\dagger}$ where:

$$
\mathbf{H}=\mathbf{\Sigma}_{\mathbf{Q}} \circ \mathbf{G} .
$$

Here, $\circ$ represents Hadamard product between $\boldsymbol{\Sigma}_{\mathbf{Q}}$, which is a $N \times N$ deterministic channel matrix, and $\mathbf{G}$ is a $N \times N$ matrix whose elements are Rayleigh distributed random variables. Matrix of this form arise in many scenarios of wireless communications [13, Th. 3.9]. Under certain conditions, $\mathbf{H H}^{\dagger}$ has a limiting eigenvalue distribution that can be approximated by a scaled version of Marčenko- Pastur Law. We also assume a modified version of Letzepis free space pathloss model for the $802.11 \mathrm{~b}$ type channel structure. For this analysis, the transmitter at index one is to be treated as reference and we consider 40 transmitters to get a channel snapshot. Fig. 3(b) and 4(b) illustrate the power attenuation due to free space pathloss for low and high ratios of channel to system bandwidth, respectively. Extending Letzepis' work, we introduce the notion of partial overlapping factor. Mishra et al. [11, Eq. 2] showed that partial overlapping phenomenon results in a decrease in received power. This is also shown in Fig. 3(a) and 4(a) for low and high ratios of channel to system bandwidth, respectively. Fig. 3(c) and 4(c) illustrate the combined effect of both the partially overlapping nature of the channel and pathloss attenuation. The deterministic matrix $\boldsymbol{\Sigma}_{\mathbf{Q}}$ is, therefore, defined as the Hadamard product of two deterministic matrices: the partial overlap channel factor matrix, $\boldsymbol{\Sigma}_{\mathrm{Q}_{\mathrm{poc}}}$ and free space pathloss channel matrix, $\Sigma_{\mathbf{Q}_{\text {pathloss }}}$. Hence,

$$
\boldsymbol{\Sigma}_{\mathrm{Q}}=\boldsymbol{\Sigma}_{\mathrm{Q}_{\text {poc }}} \circ \boldsymbol{\Sigma}_{\mathbf{Q}_{\text {pathloss }}}
$$

where $\boldsymbol{\Sigma}_{\mathbf{Q}_{\text {pathloss }}}$ and $\boldsymbol{\Sigma}_{\mathbf{Q}_{\text {poc }}}$ are circular and symmetric matrices.

$$
\overbrace{\sum_{n=n_{1}}^{n_{2}} \underbrace{\sum_{\mathbf{Q}(\mathbf{n}, \mathbf{x})}^{x_{2}}\left(\frac{a+(n-1) \tilde{C}+1-x}{a}\right)}_{\text {Inner summation }}\left(1+(x-1) \frac{D}{N}\right)^{-\frac{\lambda}{2}}}^{\text {Outer summation }}
$$

The variance profile matrix, $\boldsymbol{\Sigma}_{\mathbf{Q}(\mathbf{n}, \mathbf{x})}$ representing the partial overlapping channel model is defined as in (5). Here, $x_{1}=1+(n-1) \tilde{C}$, and $x_{2}=\min (a+(n-1) \tilde{C}, n \tilde{C})$ are the lower and upper limits of inner summation; $n_{1}=1$, and $n_{2}=N / \tilde{C}$ are the lower and upper limits of outer summation respectively; $a$ is the number of bins per channel bandwidth (1); $\lambda$ is free space pathloss exponent (magnitude two); $x$ is the transmitter indices; $D$ and $N$ are defined in Section III-A. Considering the rightside of (5), the inner summation is computed for the total number of bins within a single channel slot (Channels 1-13). We assume a linear channel assignment of $1,2,3, \ldots, 12,13,1,2,3, \ldots$ as in Section III-A. The outer sum thus repeats this 13 cell array slot over $N$ cells. (5) is the element wise representation of the matrix defined in (4), and is the product of the path loss attenuation and the partial overlap channel factor. It models the partially overlapping nature of wireless channel for an IEEE 802.11b type system with fading, and free space pathloss. Assuming that the variance profile $\boldsymbol{\Sigma}_{\mathbf{Q}}$ is circulant and symmetric, capacity can be approximated using Marčenko-Pastur Law distribution (from Letzepis [7] and Verdu [13]),

$$
\lim _{N \rightarrow \infty} \mathrm{C}_{\mathrm{opt}}\left(\mathbf{H H}^{\dagger} ; \gamma\right) \cong \mathcal{V}_{\mathrm{mpf}}\left(\boldsymbol{\Sigma}_{\mathbf{Q} \gamma}, \mathrm{K}\right)
$$

Here mpf is the Marčenko-Pastur Law function which approximates the limiting eigenvalue distribution on $\mathbf{H H}^{\dagger}, \gamma$ is SNR, and $\mathcal{V}_{\text {mpf }}(\cdot)$ is the Shannon transform of the Marčenko-Pastur Law on $\boldsymbol{\Sigma}_{\mathbf{Q}}$, respectively. $\boldsymbol{\Sigma}_{\mathbf{Q}}$ is hence computed using (5) and (6) is used to evaluate the analytical approximation for capacity using low and high values of $\gamma$.

\section{B. Monte Carlo Simulations}

The bin channel matrix $\mathbf{H}_{\tilde{\mathbf{b}}}$ is computed for individual bins. Each trial of the simulation involves constructing the $\mathbf{H}_{\tilde{\mathbf{b}}}$ matrix for $N$ cells. Capacity is evaluated by substituting this channel matrix in the log det formula (Telatar [14]). This is then summed over the total bins, $\tilde{B}$ to compute the total percell sum-rate capacity of the POC structure. Power constraint 


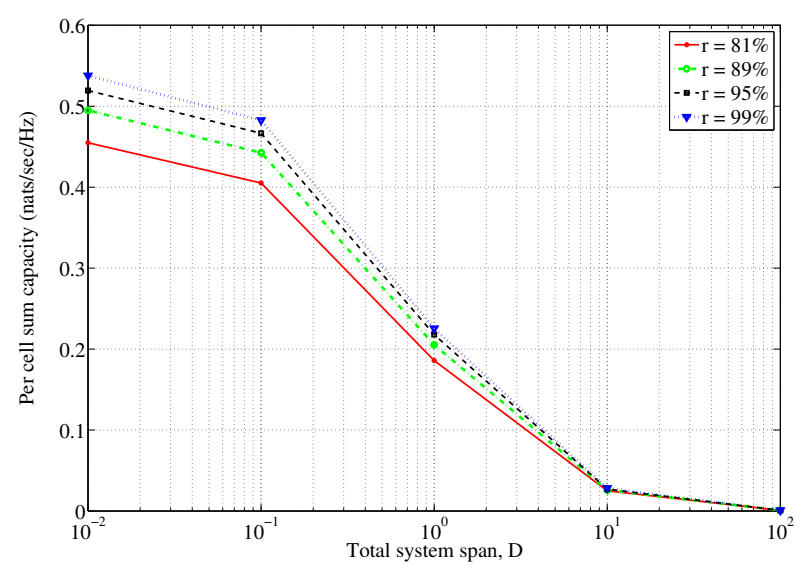

Fig. 5. Per-cell sum-rate capacity of varying-bandwidth POC for different AP density deployments $\left(\frac{B_{\text {channel }}}{B_{\text {tot }}}(\right.$ or $r$ ) in $\%$ ) for a low SNR IEEE 802.11 b type system $\left(N=100, K=1, \tilde{C}=13, B_{\text {tot }}=85 \mathrm{MHz}\right)$

is inherent for the Gaussian model, adopted by Wyner [4] and is restricted by IEEE $802.11 \mathrm{~b}$ standards. It is hence considered in our analysis. The model exploits the fact that bin power remains constant over $B_{\text {channel. }}$. Therefore, transmitted power over the channel bandwidth defined as $P_{\tilde{c}}$ can be represented mathematically as,

$$
P_{\tilde{c}}=\sum_{\tilde{b}=\tilde{c}}^{\tilde{c}+\frac{B_{\text {channel }}}{F_{\text {bin }}}-1} P_{\tilde{b}, \tilde{c}}
$$

where $P_{\tilde{b}, \tilde{c}}$ is the average transmitted power in $\tilde{b}$ bin for a given channel, $\tilde{c}$. The per bin sum capacity can then be represented using Cover [15] and Telatar [14] as:

$$
C_{\text {bin }}=\log \operatorname{det}\left(\mathbf{I}_{\tilde{\mathbf{b}}}+\frac{P_{\tilde{b}, \tilde{c}} \cdot \mathbf{H}_{\tilde{\mathbf{b}}} \mathbf{H}_{\tilde{\mathbf{b}}}^{\dagger}}{N_{o} \cdot F_{b}}\right)
$$

We also know that,

$$
\frac{P_{\tilde{b}, \tilde{c}} \cdot \tilde{B}}{N_{0} \cdot F_{b} \cdot \tilde{B}} \equiv \frac{P_{\tilde{c}}}{\sigma_{N}^{2}}
$$

where $\sigma_{N}^{2}$ is the channel noise power and $P_{\tilde{c}}$ is the maximum channel transmit power. Finally, summing over all the bins within the bandwidth,

$$
C_{\text {partial overlap }}=\sum_{\tilde{b}=1}^{\tilde{B}} \log \operatorname{det}\left(\mathbf{I}_{\tilde{\mathbf{b}}}+\frac{P_{\tilde{c}}}{\sigma_{N}^{2}} \cdot \mathbf{H}_{\tilde{\mathbf{b}}} \mathbf{H}_{\tilde{\mathbf{b}}}^{\dagger}\right)
$$

where $\mathbf{H}$ is the Hermitian matrix and $\mathbf{H}^{\dagger}$ is its conjugate transpose; $\sigma_{N}^{2}$ is the noise power spread over each frequency bin and $P_{\tilde{b}, \tilde{c}}$, is the average transmitted power per bin for channel, $\tilde{c}$.

\section{RESUlts}

\section{A. Impact of AP density and bandwidth utilization on POC capacity}

Fig. 5 shows the effect of AP density on per-cell sum-rate capacity of a POC based system. The effect of bandwidth

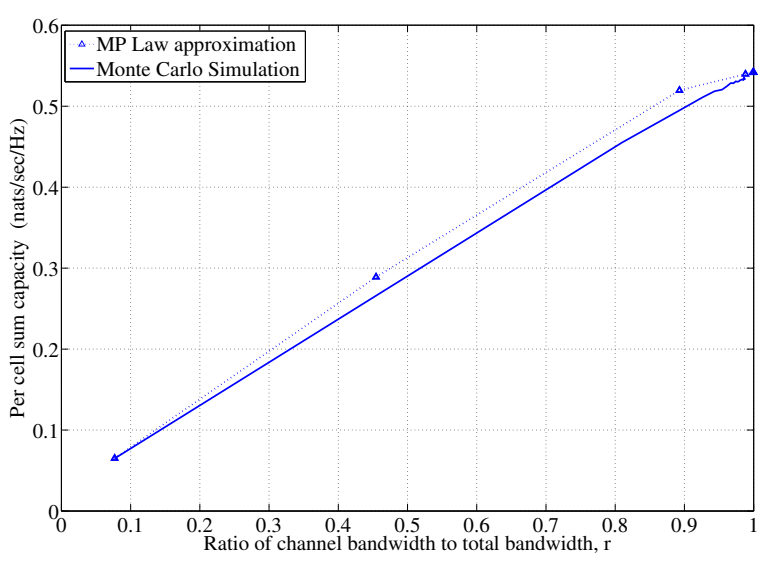

Fig. 6. Per-cell sum-rate capacity of POC type systems for different bandwidth ratios $(r)$ for a low SNR flat-fading IEEE 802.11b type system $\left(N=100, D=10^{-2}, K=1, \gamma=0.01 \mathrm{~dB}\right)$.

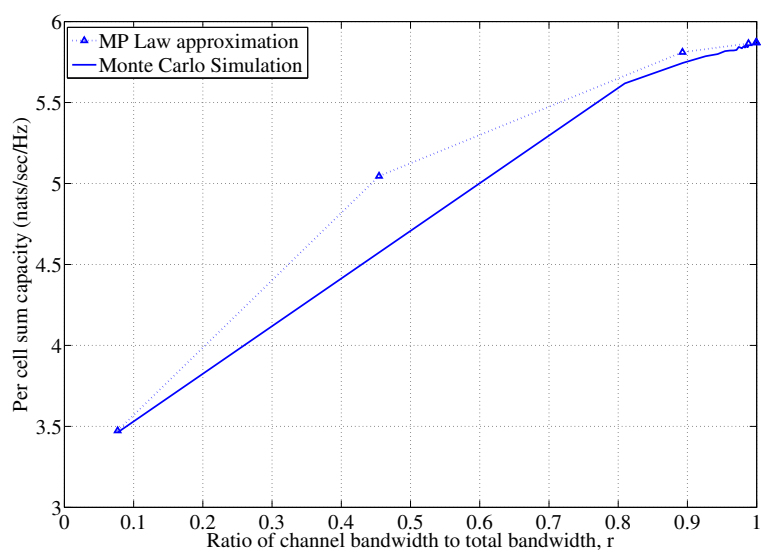

Fig. 7. Per-cell sum-rate capacity of POCtype systems for different bandwidth ratios (or $r$ ) for a high SNR flat-fading IEEE 802.11b type system $\left(N=100, D=10^{-2}, K=1, \gamma=10 \mathrm{~dB}\right)$.

variation, $r$ is clearer in high density AP deployments. This can be explained due to the multiplicative effect of the path loss and POCs on channel gain. It attenuates the signal sharply over a smaller distance; thus, smaller distances between APs will result in better performance. Here, the difference between capacity due to orthogonal and fully overlapping channels increases to 0.1 nats $/ \mathrm{sec} / \mathrm{Hz}\left(D=10^{-2}\right)$. At this density, a $4 \%$ reduction in channel bandwidth (decrease from $99 \%$ to $95 \%$ of max. bandwidth) reduces the per-cell sum-rate capacity by only $2.6 \%$. From the above and Fig. 5, we can hence deduce that for dense AP deployments, a channel overlap of approximately $90 \%$ and more results in a near-optimal data rate for POC type systems.

\section{B. POC capacity approximation and simulation}

The results of this section are summarized in Fig. 6 and 7. These figures show the difference in per-cell sum-rate capacity of fading channel for low $\operatorname{SNR}(\gamma=0.01 \mathrm{~dB})$, as 
in Fig. 6 and a high SNR $(\gamma=10 \mathrm{~dB})$ as in Fig. 7, when optimum joint decoding is performed using hyper-receiver for partially overlapping channels. Here $N=100, D=10^{-2}$ and $K=1$. In each figure, capacity is approximated for each value of $r$. Monte Carlo Simulations illustrate the validity of the approximations which are performed in (6). To compare the validity of the approximation, the channel to system bandwidth ratio $r$, is varied. For each corresponding value of $a$, a number of trials are done, and in each trial,a new $\mathbf{H}$ is constructed for $N=100, K=1, D=10^{-2}$, using the variance profile, $\boldsymbol{\Sigma}_{\mathbf{Q}}$. At each trial, $\mathbf{H}_{\tilde{\mathbf{b}}}$ is constructed and used to computed the function $\log \operatorname{det}\left(\mathbf{I}_{\mathrm{N}}+\gamma_{\tilde{b}} \cdot \mathbf{H}_{\tilde{\mathbf{b}}} \mathbf{H}_{\tilde{\mathbf{b}}}^{\dagger}\right)$. Here $\gamma_{\tilde{b}}$ is $\frac{P_{\tilde{c}}}{\sigma_{N}^{2}}$ as in (10). Simulation conform well to approximation with a mean error of approximately $5 \%$. We observe that for a fixed $B_{\text {tot }}$, the higher the channel bandwidth, the larger is the capacity. Considering the Monte Carlo simulation (in bold) for low SNR region of the partial overlapping channel capacity (Fig. 6). In the region of lower channel to total bandwidth usage, capacity is small. This is because of the low SNR and small interference (fewer overlapping channels). As the channel bandwidth becomes large, power gain due to multiple channels increases the capacity. The number of interfering bins increases linearly. When the bins per channel approach 0.9 , the effect of partial overlap factor is negligible; see Fig. 4(c). Adjacent channel interference caused by POC structure, counters the increase due to power gain. The capacity saturates in the presence of nearly full overlap. The overlapping channel model thus reduces to that of [7]. In addition to, Fig. 7 for high SNR region of POC type system, in the region of low channel to total bandwidth utilization, capacity increases linearly. The high SNR results in a large value for small overlap. However, as the number of interfering bin increases, the power gain more than compensates for the adjacent channel interference. As the channel to total bandwidth ratio approaches 0.5 , the slowly increasing level of adjacent channel interference overcomes the power gain advantage. This effect saturates the capacity and the model reduces to that of [7] when $r=1$.

\section{CONCLUSIONS AND FUTURE WORK}

We have analyzed the capacity of linearly distributed APs utilizing POC structure as in WLAN (IEEE 802.11b). Wyner's GCMAC [4] with flat fading and free space pathloss was used. Under our proposed model, bandwidth is partitioned into bins and capacity for each bin is computed and summed over the entire bandwidth. For performance evaluation, we considered approximation based on Marčenko-Pastur Law. This was implemented by combining the effect of POC factor and pathloss attenuation. Approximations were computed for low and high values of SNRs. We validated our approximations with Monte Carlo simulations using a derived logdet formula. The simulations conform to analysis with an approximate mean error of $5 \%$. We further explored the effect of AP density and found that POC based systems performed well in high density deployments. We computed capacity for a range of overlaps and concluded that near optimum data rate is achievable for a channel overlap of $90 \%$ and above.
A linear assignment of frequency channels is assumed for this paper. Further, single user per cell is also assumed. These are simplified assumptions which will be extended to include user distribution and channel assignment for a more realistic analysis of POC-type systems.

\section{ACKNOWLEDGMENT}

The authors would like to acknowledge Dr. Costas Tzaras for his contributions to this paper.

\section{REFERENCES}

[1] A. Mohamed, "Welcome to the wireless city," Computer Weekly, 13 February 2007. [Online]. Available: www.computerweekly.com

[2] J. Pinkney and A. Sesay, "Characterization of the on-body wireless channel at 2.4 and $5.8 \mathrm{GHz}$," in Vehicular Technology Conference, 2005. VTC-2005-Fall. 2005 IEEE 62nd, vol. 2, 25-28 Sept., 2005, pp. 12941298.

[3] "Channel Deployment Issues for 2.4-GHz 802.11 WLANs," Cisco Systems Inc., Tech. Rep., 2004.

[4] A. Wyner, "Shannon-theoretic Approach to a Gaussian Cellular Multiple-Access Channel," Information Theory, IEEE Transactions on, vol. 40, no. 6, pp. 1713-1727, Nov. 1994.

[5] M. Clark, K. Leung, B. McNair, and Z. Kostic, "Outdoor IEEE 802.11 cellular networks: radio link performance," in Communications, 2002. ICC 2002. IEEE International Conference on, vol. 1, 28 April-2 May 2002, pp. 512-516.

[6] O. Somekh and S. Shamai, "A Shannon-theoretic view of Wyner's multiple-access cellular channel model in the presence of fading," Information Theory, 1998. Proceedings of IEEE International Symposium on, p. 393, 16-21 Aug. 1998.

[7] N. Letzepis, "Gaussian cellular multiple access channels," Ph.D. dissertation, Institute of Telecommunications Research, University Of South Australia, December 2005.

[8] H. Liu, H. Yu, X. Liu, C.-N. Chuah, and P. Mohapatra, "Scheduling multiple partially overlapped channels in wireless mesh networks," in Communications, 2007. ICC '07. IEEE International Conference on, 24-28 June 2007, pp. 3817-3822.

[9] A. H. M. Rad and V. W. S. Wong, "Partially Overlapped Channel Assignment for Multi-Channel Wireless Mesh Networks," in Communications, 2007. ICC 'O7. IEEE International Conference on, 24-28 June 2007, pp. 3770-3775.

[10] A. Naveed, A. Naveed, S. S. Kanhere, and S. K. Jha, "Topology control and channel assignment in multi-radio multi-channel wireless mesh networks," in Proc. IEEE Internatonal Conference on Mobile Adhoc and Sensor Systems MASS 2007, S. S. Kanhere, Ed., 2007, pp. 1-9.

[11] A. Mishra, V. Shrivastava, S. Banerjee, and W. Arbaugh, "Partially overlapped channels not considered harmful," SIGMETRICS Perform. Eval. Rev., vol. 34, no. 1, pp. 63-74, 2006.

[12] R. Akl and A. Arepally, "Dynamic Channel Assignment in IEEE 802.11 Networks," Portable Information Devices, 2007. PORTABLE07. IEEE International Conference on, pp. 1-5, 25-29 May 2007.

[13] A. M. Tulino and S. Verdu, Random matrix theory and wireless communications. Hanover, MA, USA: Now Publishers Inc., 2004.

[14] I. E. Telatar, "Capacity of Multi-antenna Gaussian Channels," European Transactions on Telecommunications, vol. 10, no. 6, pp. 585-595, November 1999.

[15] T. M. Cover and J. A. Thomas, Elements of Information Theory, Second ed. John Wiley \& Sons, 2006. 